\title{
Modelling of linear test bench for short distance measurements
}

\author{
Lauryna Šiaudinytè \\ Institute of Geodesy, Vilnius Gediminas Technical University, Saulètekio al. 11, LT-10223, Vilnius, Lithuania
}

\begin{abstract}
To perform the calibration of total stations under laboratory conditions, lack of space is a common issue. The paper presents the analysis of applied solutions for short distance measurements and assumptions of the construction development of a linear test bench for cyclic error determination. Various aspects of improvement are explained in the paper, and the preliminary design of a linear test bench being developed at Vilnius Gediminas Technical University is presented.
\end{abstract}

\section{Section: RESEARCH PAPER}

Keywords: Calibration bench; laser interferometer; linear measurements

Citation: Lauryna Šiaudinytė, Modelling of linear test bench for short distance measurements, Acta IMEKO, vol. 4, no. 2, article 12, June 2015, identifier: IMEKO-ACTA-4 (2015)-02-12

Editor: Paolo Carbone, University of Perugia, Italy

Received October 15, 2014; In final form November 27, 2014; Published June 2015

Copyright: () 2015 IMEKO. This is an open-access article distributed under the terms of the Creative Commons Attribution 3.0 License, which permits unrestricted use, distribution, and reproduction in any medium, provided the original author and source are credited

Funding: This work was supported by the European Social Fund under the Global Grant measure

Corresponding author: Lauryna Šiaudinytė, e-mail: lauryna.siaudinyte@vgtu.It

\section{INTRODUCTION}

The main standard dealing with total stations testing is ISO 17123. However, this standard focuses on field testing procedures. Although a total station is mainly intended for outdoor measurements, indoor calibration in controlled environmental conditions provides the best way to determine systematic errors. However, testing devices under laboratory conditions usually come with new issues of which lack of space is typically one of the most important. This brings to the need of test bench development for the calibration of geodetic distance measuring equipment within relatively short distances.

In many calibration laboratories there are special benches designed for tape calibration which could be adjusted for electronic distance measuring equipment calibration. Section 2 reveals applied instrumentation for indoor distance measurements of various calibration laboratories.

Proposed possibilities for the development of a short distance linear calibration bench using the existing basis of special supports for cyclic error determination are presented further in this paper. The new calibration bench of aluminium construction is cost saving and lightweight compared to stainless steel. Within 13 meters of the bench it is possible to determine systematic errors and therefore to increase accuracy.
Automation of the carriage will reduce the measurement uncertainty caused by human interaction.

\section{DISTANCE MEASURING INSTRUMENTATION}

Preservation of the primary length standard - the meter - is the main role of length metrology. It also has to ensure the infrastructure needed for dimensional and positional measurements traceability to the meter.

The meter is defined as the length of the path traveled by light in vacuum during the time interval of 1/299 792458 of a second. An interferometer is an instrument providing standard distance measurements. There are a few main types of laser interferometers used in laboratory measurements - homodyne and heterodyne laser interferometers are the most commonly used. Homodyne interferometers are based on the interference of two beams (one split beam) of the same frequency. The optics of homodyne interferometers is similar to Michelson interferometers, however it produces a lower signal due to a high noise ratio compared to the heterodyne interferometer. Heterodyne interferometers are based on two beams with different frequencies (low and high), different polarization mixed with each other and in non-linear combination creating two new frequencies (heterodynes). The double frequency 


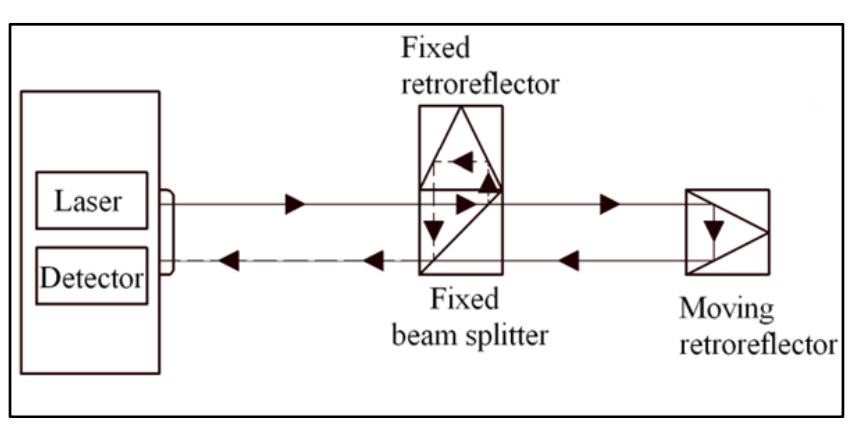

Figure 1 . Working principle of a laser interferometer.

interferometers measure the relative displacement of two reflectors by splitting the beam.

Then two beams are directed to different retro-reflectors and the resulting signals are returned to a photodetector [7]. The working principle of the laser interferometer is shown in Figure 1. Heterodyne interferometers are very sensitive and usually more accurate than homodyne interferometers.

There are environmental factors such as temperature, pressure and humidity influencing linear measurements. The most important factor of length measurement accuracy is temperature. The sensitivity of interferometric systems to varying temperature can significantly influence the measurement results because of linear expansion of materials affecting the stability of the structural geometry. Precision measurement systems are usually placed in a controlled environment with a constant temperature of $20.0{ }^{\circ} \mathrm{C} \pm 0.2{ }^{\circ} \mathrm{C}$. To meet such requirements the interferometric system should be placed in a special chamber with many parameter measuring sensors to maintain an effective thermal and pressure isolation. These sensors as well as temperature fluctuation can bring in the uncertainty in such precise length measurements [3]. Another important uncertainty source in dimensional metrology is the abbe error which occurs due to the difference between the axis of the measurement object and the axis of the measuring device. This error can also be caused by the motion of the measurement object [6].

\subsection{Applied solutions for indoor calibration}

In the National Metrology Institute of Germany (Physikalisch-Technische Bundesanstalt) there is a $50 \mathrm{~m}$ long geodetic base which is also a comparator established for the calibration of length measuring instruments where the principle of Michelson's interferometer is used as a length standard. The carriage is mounted on the rails fixed along the $50 \mathrm{~m}$ base. The stationary part of an interferometer is placed on a stable base in front of the rails. The laser beam is split into two paths generated by a reflector consisting of three mirrors arranged on the surface of a cube, and fixed to the moving carriage. The zero point of the interferometer in respect of the rangefinder is determined by means of a gauge block. The measurement error of the distance measuring device can be determined by comparison with the distance measured by an interferometer and a calibrated instrument [10]. In KRISS the $50 \mathrm{~m}$ long bench was developed for the calibration of surveying tapes. The main components of this system are the interferometer and the carriage moved by the servo motor attached to it along steel rods and controlled by a computer through wireless communication. The interferometer axis is set in the center between the rails where the tape is placed. There is an optical system with the microscope installed in the moving carriage to detect the graduation of the tape. The distance measured by the interferometer is compared to the distance determined by the tape being calibrated [4].

$50 \mathrm{~m}$ long benches are very common in metrology institutes, however, such distance may cause additional uncertainty. It is a challenging task to align all the components and install the precision rail system while avoiding inclination and geometric alterations due to thermal expansion or compression.

Therefore, the Finish Centre for metrology and accreditation developed a shorter $-30 \mathrm{~m}$ long distance calibration bench. For stability this bench was built (constructed) two floors under the ground. Concrete pillars are placed at every $2.8 \mathrm{~m}$ and a $31.5 \mathrm{~m}$ monolithic concrete beam on top of them. There are two separate bases established in both ends of the bench for the reference equipment and instruments under calibration. The rails are mounted on additional supports and the carriage moving along the rail is equipped with laser interferometer optics for both distance and angle measurements as well as a microscope with CCD camera for linear position detection. This interferometric bench is suitable for a laser tracker, a total station and tape calibration. The expanded uncertainty $(k=2)$ of the length scale is $2.6 \cdot 10^{-6} \mathrm{~m}$ for a $30 \mathrm{~m}$ displacement [9].

Although smaller benches can fit laboratory premises better, there is a need to calibrate total stations in longer distances because they are designed to be used outdoors by measuring longer stretches. To extend measuring distances in the laboratory a special mirror system can be used. To double or triple the measuring distance a zigzag path can be created for the beam to travel by using mirrors mounted on certain points and rotated at a certain angle. Such method allows avoiding reflector mounting outside the laboratory [5].

Due to the space issue at Vilnius Gediminas Technical University it was decided to create a $13 \mathrm{~m}$ long linear bench for short distance measurements and cyclic periodic error determination.

\section{THE LINEAR BENCH}

The linear bench under development at the Institute of Geodesy of the Vilnius Gediminas Technical University is suitable for the calibration of geodetic electro-optical equipment. The main instrument used in survey is a total station. This instrument is exceptional because of its ability to measure horizontal and vertical angles as well as distances simultaneously. An Electronic Distance Meter (EDM) is embedded in the total station for the distance measurements. This instrument is typically based on the phase difference measuring principle for distance measurements. The measuring signal modulated on the carrier wave in the emitter travels a distance $\mathrm{D}$ from the total station $\mathrm{A}$ to reflector $\mathrm{B}$ and back to the instrument receiver. In the receiver the difference between transmitted and received signals are measured and compared. Point $\mathrm{A}$ and point $\mathrm{A}^{\prime}$ shown in Figure 2 are the same however the returned wave has been opened out. In an EDM the double distance length (2D) is measured by a difference in phase angles of two sinusoidal waves (transmitted and received). This distance can be expressed in terms of wavelength of the measuring unit [8]:

$$
2 D=n \lambda_{m}+\Delta \lambda_{m}
$$

where $\lambda_{\mathrm{m}}$ is the wavelength of the measuring unit, $n$ is an integer number of wavelengths travelled by the wave, $\Delta \lambda_{m}$ is a fraction of the wavelength travelled by the wave. 


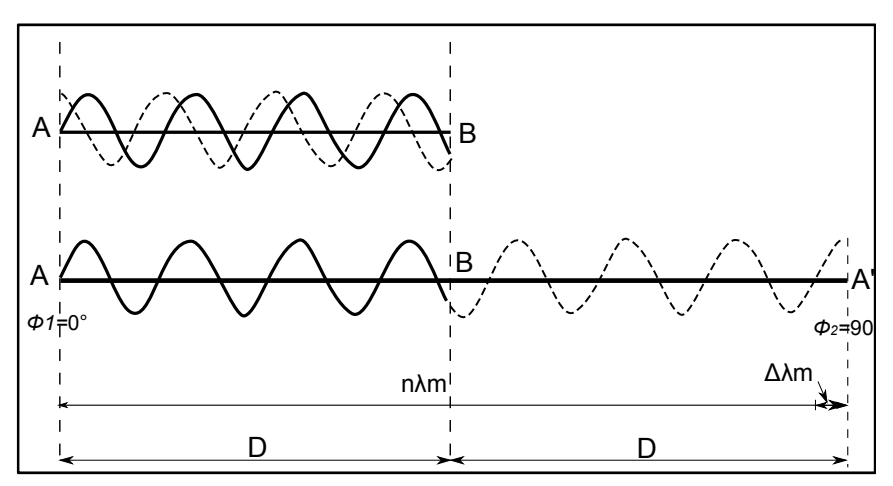

Figure 2. Principle of phase shift measurement.

The fraction of the wavelength travelled by the wave $\left(\Delta \lambda_{\mathrm{m}}\right)$, when the phase angle of the transmitted wave is $\varphi_{1}$ and the phase angle of the received wave is $\varphi_{2}$, is expressed as follows:

$\Delta \lambda_{m}=\frac{\left(\varphi_{2}-\varphi_{1}\right)}{2 \pi} \lambda_{m}$

Deterioration of the inner system components over time can influence the measurement results. Therefore, instruments have to be tested regularly to determine short periodic or cyclic errors. The cyclic error is sinusoidal with a wavelength equal to the unit length of the instrument.

\subsection{The former stand for cyclic error determination}

A $15 \mathrm{~m}$ geodetic baseline for EDM short distance calibration at the Vilnius Gediminas Technical University was established in collaboration with the Finish Geodetic Institute in 2001. In total 16 stands were attached to the grooved profile which was fixed to a stabilized wall. One stand was established especially for EDM mounting and the other 15 for reflector mounting. All the stands were aligned, levelled and established at a pitch of $1 \mathrm{~m}$.

The basement location of this laboratory led to a stable construction of this bench. After establishment this bench was calibrated by the Finish Geodetic Institute. A Wild Distomat DI 2002 and a Wild Theomat T2002 tachometer along with a Wild GPH1AP reflector were calibrated at the Nummela Standard Baseline and used for distance measurements. In total 70 lengths were measured performing repetitive measurements from stands $0,2,5,7,8$. Calibration results are shown in Table 1.

Table 1. The results of distance measurements between the stands.

\begin{tabular}{|c|c|}
\hline $\begin{array}{c}\text { Stand } \\
\text { number }\end{array}$ & $\begin{array}{c}\text { Measurement result } \\
\mathbf{Y} \pm \mathbf{U}_{95 \%} \mathbf{( k = 2 )}\end{array}$ \\
\hline $0-1$ & $1000.2 \mathrm{~mm} \pm 0.5 \mathrm{~mm}$ \\
\hline $0-2$ & $2000.6 \mathrm{~mm} \pm 0.2 \mathrm{~mm}$ \\
\hline $0-3$ & $3001.4 \mathrm{~mm} \pm 0.3 \mathrm{~mm}$ \\
\hline $0-4$ & $4001.4 \mathrm{~mm} \pm 0.4 \mathrm{~mm}$ \\
\hline $0-5$ & $4999.2 \mathrm{~mm} \pm 0.3 \mathrm{~mm}$ \\
\hline $0-6$ & $5999.2 \mathrm{~mm} \pm 0.5 \mathrm{~mm}$ \\
\hline $0-7$ & $7000.8 \mathrm{~mm} \pm 0.3 \mathrm{~mm}$ \\
\hline $0-8$ & $7997.9 \mathrm{~mm} \pm 0.3 \mathrm{~mm}$ \\
\hline $0-9$ & $9000.8 \mathrm{~mm} \pm 0.5 \mathrm{~mm}$ \\
\hline $0-10$ & $10001.0 \mathrm{~mm} \pm 0.5 \mathrm{~mm}$ \\
\hline $0-11$ & $10999.2 \mathrm{~mm} \pm 0.5 \mathrm{~mm}$ \\
\hline $0-12$ & $12000.4 \mathrm{~mm} \pm 0.5 \mathrm{~mm}$ \\
\hline $0-13$ & $12998.9 \mathrm{~mm} \pm 0.5 \mathrm{~mm}$ \\
\hline $0-14$ & $14000.4 \mathrm{~mm} \pm 0.5 \mathrm{~mm}$ \\
\hline $0-15$ & $15005.1 \mathrm{~mm} \pm 0.5 \mathrm{~mm}$ \\
\hline
\end{tabular}

These indoor facilities can be used for all electronic distance measuring equipment. The EDM of laser scanners are also calibrated using this cyclic error determination baseline. The target is placed on the established stands and scanned. Each scanned target consists of a cloud of approx. 39000 points which are used to determine the coordinates of the target centres. Then the measured distances are compared with the calibrated distances between the cyclic error determination baseline stands [1].

\subsection{The linear bench under development}

Distance measurement equipment has long been tested and controlled by implementing non automated measurements and measuring objects at certain well-known distances. At the Institute of Geodesy such principle has been used by implementing multiple fixed indoor stands for short distance measurements and cyclic error correction, as well as several fixed outdoor pillars were used for long distance measurement control. Nonetheless, implementation of such instrumentation requires a lot of human interaction in the process which slows down the process considerably and introduces multiple operator related errors. Additionally, since modern electronic measurement equipment (distance meters, total stations etc.) can determine an enormous amount of measurement data with very high resolution, the implementation of fixed pillars for error determination is not fully correct. The distances between fixed stands might include possible systematic errors which are left uncovered by such a calibration. The issues like the calibration step under laboratory conditions are neither covered in the international standards (i.e. ISO).

Implementation of automated measurement and control of the process would allow to increase both the speed and the accuracy of the measuring process. However, automated systems are available mostly for short distances. Although the mentioned automation brings in lots of benefits to the measurement process, it still remains costly, complex and not always affordable for some organizations. Therefore, the Institute of Geodesy is developing a linear test bench consisting of standard components and cost effective aluminum profiles.

The test bench implements the distance meters' cyclic correction stand composed of multiple firmly mounted supports as the base for the linear movement rail. The rail itself is a standard aluminum profile made for steel roller bearing linear guides to roll the mounted moving car. Implementation of aluminum profiles ensures a lower total mass relevant due to implementation of inherited components of the base. However, some inaccuracies due to the lower stiffness are unavoidable. The profile rail is connected supports (with a step of 2 meters) and precisely levelled. Preliminary design of the linear calibration bench and its components $(\mathrm{a}$ - moving carriage, $\mathrm{b}-$ rails, c - supports, d - reflector for total station measurements, e - corner cube fixed to the moving carriage to create the path for the split laser beam, 1 - distance between the firmly mounted supports, 1 meter) is shown in Figure 3.

The moving carriage of the bench is an aluminum body plate moving on precise ball bearings. A motorized linear air bearing would be the main component ensuring a smooth motion of the carriage. Therefore, vertical and horizontal straightness as well as rotational motion errors have to be determined before using the carriage for the calibration of length measuring instruments [2].

Implementation of aluminum here is preferable due to the decrease of moving mass its inert influence on the rail profile. 


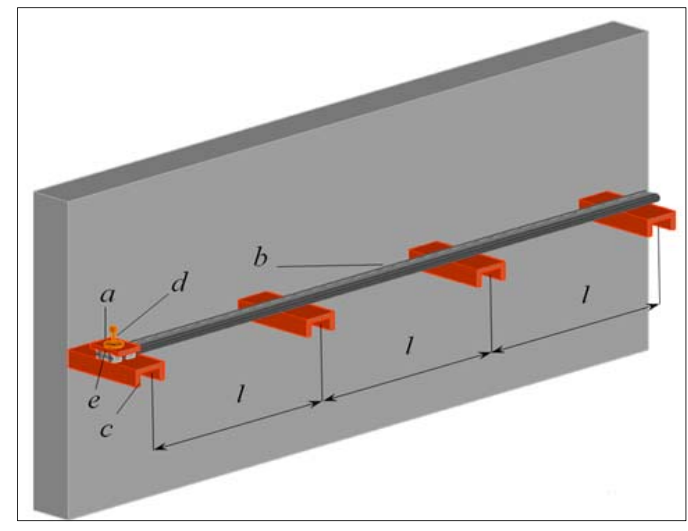

Figure 3. Preliminary design of the linear bench.

The movement of the car is automated by step motors (with coarse distance determination) and the car can be positioned at practically any linear position within the distance of the bench. The precise position of the car is determined by a stationary fixed Renishaw interferometer with its mirror mounted on the moving car.

For the uncertainty evaluation it is important to consider the following aspects. Since the aluminum profile is supported at a pitch of $1 \mathrm{~m}$, the deflection of the profile is $0.03 \mathrm{~mm}$ in every stretch with a bending stress of $0.74 \mathrm{~N} / \mathrm{mm}^{2}$ because of both carriage and reflector weights, which is up to $10 \mathrm{~kg}$. EMD measurements are mostly affected by environmental conditions which can be minimized by performing measurements in a laboratory. However, stabilizing the temperature and atmospheric correction is obligatory according to ISO 17123:5 because temperature, pressure and humidity affect the velocity of the signal. It is also very important to perform slope and zero point corrections. Zero point errors are related to electrical delays, the differences between the electronic and mechanical center of the instrument. The scale error which is caused by internal frequency errors also has an impact on measurement results. The abbe error is one of the main error sources in these linear measurements because of nonlinearity of the carriage base plate and the rails. To sum up, the alignment of reflector and total station, abbe error and the motion of the carriage are the main uncertainty sources. So far the research showed that an expanded uncertainty of $3.0^{-1} \cdot 10^{-6} \mathrm{~m}$ for $13 \mathrm{~m}$ displacement can be achieved, however, the system needs to be stabilized and further multiple measurements in various displacements will be provided with mentioned uncertainty components in further research.

\section{CONCLUSIONS}

The assumptions of using fixed cyclic error determination stands as a base for the development of a linear calibration bench with an automated moving carriage are presented in this paper. The advantages of this proposed bench are the lightweight of the used materials as well as a cost saving solution. However, it is more complicated to precisely control the environment where the calibration is performed.

The main purpose of this linear bench is to reduce human interaction in the process and to determine cyclic and other distance measurement errors by performing measurements at a desired distance within the length of the bench.

\section{ACKNOWLEDGEMENT}

This research is funded by the European Social Fund under the Global Grant measure (project No. VP1-3.1-ŠMM-07-K01-102).

\section{REFERENCES}

[1] U. Antanavičiūtè, R. Obuchovski, E. Paršeliūnas, D. Popovas, D. Ślikas, "Some issues regarding the calibration of the terrestrial laser scanner Leica Scanstation C10", in: Geodesy and Cartography, Vol. 39(3), 2013, pp. 138-143.

[2] W. Gao, Y. Arai, A. Shibuya, S. Kiyono, C. H. Park, "Measurement of multi-degree-of-freedom error motions of a precision linear air-bearing stage", in: Precision Engineering, Vol. 30, Issue 1, 2006, pp. 96-103.

[3] R. Hamid, D. Sendogdu, C. Erdogan, "The temperature stabilization and temperature measurement of a Kösters interferometer", in: Measurement Science and Technology, 2005. Vol. 16, p. 2201-2207.

[4] J.W. Kim, D. Y. Jeong, M. S. Kim, C. S. Kang, J. A. Kim, T. B. Eom, "A $50 \mathrm{~m}$ laser interferometer for automatic calibration of surveying tapes using wireless communication" in: Measurement Science and Technology, 2008, Vol 19.

[5] R. Khalil, "New Compact method for laboratory testing EDM instruments", Proc. of FIG Working week 2005 and $8^{\text {th }}$ international conference on the global spatial data infrastructure, April 16-21, 2005, Cairo, Egypt, pp. 1-8.

[6] R. Köning, J. Flügge, H. Bosse, "A method for the in situ determination of Abbe errors and their correction", in: Measurement Science and Technology, 2007, Vol 18, p. 476-481.

[7] R. Paschotta, "Optical Heterodyne Detection", in: RP Photonics Consulting GmbH papers, 2012.

[8] W. F. Price, J. Uren. Laser surveying. London: Van Nostrand Reinhold (1989), ISBN 0747600236, 256p.

[9] J. Unkuri, A. Rantanen, J. Manninen, V.P. Esala, A. Lassila, "Interferometric $30 \mathrm{~m}$ bench for calibrations of 1D scales and optical distance measuring instruments", Measurement science and technology Vol. 23, 2012, pp. 1-8.

[10] M. Wedde, K. Meiners-Hagen, A. Abou-Zeid,

"Interferometrische Kalibrierung von Strichmaßen, Laserinterferometern und Entfernungsmessgeräten: Die Geodätische Basis der PTB”, in: PTB-Mitteilungen, Vol 120, 2010 . 